\title{
Portability of the thiolation domain in recombinant pyoverdine non-ribosomal peptide synthetases
}

\author{
Mark J. Calcott ${ }^{1}$ and David F. Ackerley ${ }^{1,2,3^{*}}$
}

\begin{abstract}
Background: Non-ribosomal peptide synthetase (NRPS) enzymes govern the assembly of amino acids and related monomers into peptide-like natural products. A key goal of the field is to develop methods to effective recombine NRPS domains or modules, and thereby generate modified or entirely novel products. We previously showed that substitution of the condensation (C) and adenylation (A) domains in module 2 of the pyoverdine synthetase PvdD from Pseudomonas aeruginosa led to synthesis of modified pyoverdines in a minority of cases, but that more often the recombinant enzymes were non-functional. One possible explanation was that the majority of introduced $C$ domains were unable to effectively communicate with the thiolation $(T)$ domain immediately upstream, in the first module of PvdD.

Results: To test this we first compared the effectiveness of C-A domain substitution relative to T-C-A domain substitution using three different paired sets of domains. Having previously demonstrated that the PvdD A/T domain interfaces are tolerant of domain substitution, we hypothesised that T-C-A domain substitution would lead to more functional recombinant enzymes, by maintaining native T/C domain interactions. Although we successfully generated two recombinant pyoverdines, having a serine or a N5-formyl-N5-hydroxyornithine residue in place of the terminal threonine of wild type pyoverdine, in neither case did the T-C-A domain substitution strategy lead to substantially higher product yield. To more comprehensively examine the abilities of non-native $T$ domains to communicate effectively with the $C$ domain of PvdD module 2 we then substituted the module $1 \mathrm{~T}$ domain with 18 different $T$ domains sourced from other pyoverdine NRPS enzymes. In 15/18 cases the recombinant NRPS was functional, including 6/6 cases where the introduced T domain was located upstream of a $C$ domain in its native context.
\end{abstract}

Conclusions: Our data indicate that $\mathrm{T}$ domains are generally able to interact effectively with non-native $\mathrm{C}$ domains, contrasting with previous findings that they are not generally portable upstream of epimerisation (E) or thioesterase (TE) domains. This offers promise for NRPS recombination efforts, but also raises the possibility that some $\mathrm{C}$ domains are unable to efficiently accept non-native peptides at their donor site due to steric constraints or other limitations.

\footnotetext{
* Correspondence: david.ackerley@vuw.ac.nz

${ }^{1}$ School of Biological Sciences, Victoria University of Wellington, Wellington, New Zealand

${ }^{2}$ Centre for Biodiscovery, Victoria University of Wellington, Wellington, New Zealand

Full list of author information is available at the end of the article
}

C Biomed Central

(c) 2015 Calcott and Ackerley. Open Access This article is distributed under the terms of the Creative Commons Attribution 4.0 International License (http://creativecommons.org/licenses/by/4.0/), which permits unrestricted use, distribution, and reproduction in any medium, provided you give appropriate credit to the original author(s) and the source, provide a link to the Creative Commons license, and indicate if changes were made. The Creative Commons Public Domain Dedication waiver (http://creativecommons.org/publicdomain/zero/1.0/) applies to the data made available in this article, unless otherwise stated. 


\section{Background}

Non-ribosomal peptide synthetases (NRPSs) produce a wide range of small peptide products of biotechnological interest. The peptide products can contain a highly diverse array of amino acids or related monomers, with over 600 different monomers having been detected in non-ribosomal peptides [1]. The diversity in monomers and peptide structure contributes to a wide range of bioactivities across all non-ribosomal peptides; for example, cytotoxic activities of the medically relevant peptides penicillin, daptomycin and cyclosporin A [2]. NRPSs synthesise peptides in an assembly line-like manner [3], with distinct modules governing the stepwise incorporation of specific monomers into the final product. The biosynthetic logic of this process is covered in-depth by many reviews $[2,4-6]$ and is summarised in Figure 1.

The modular nature of the NRPS assembly line offers promise for the creation of rationally modified peptides, or even combinatorial libraries of novel products, by recombining NRPS domains or modules at a genetic level. The primary substrate-specifying role of the A domain has made this domain a key target for NRPS recombination experiments. However, efforts to generate novel peptide products by modifying A domain substrate specificity, or by substituting in a non-synonymous A domain (that is, one which specifies an alternative monomer to the A domain it is replacing) have generally been unsuccessful $[7,8]$. Numerous in vitro e.g., $[9,10]$ and in vivo $[11,12]$ studies have demonstrated that the $\mathrm{C}$ domain typically "proof-reads" the downstream acceptor substrate (Fig. 1d) in a manner that prevents incorporation of nonsynonymous monomers into the peptide product. Structural studies have also indicated that disruption of the native C/A domain interface during $\mathrm{A}$ domain substitution may further impair the activity of recombinant NRPS modules [13]. Nonetheless, we found that five out of five

\section{(a)}

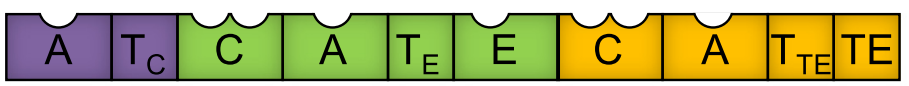

(b)
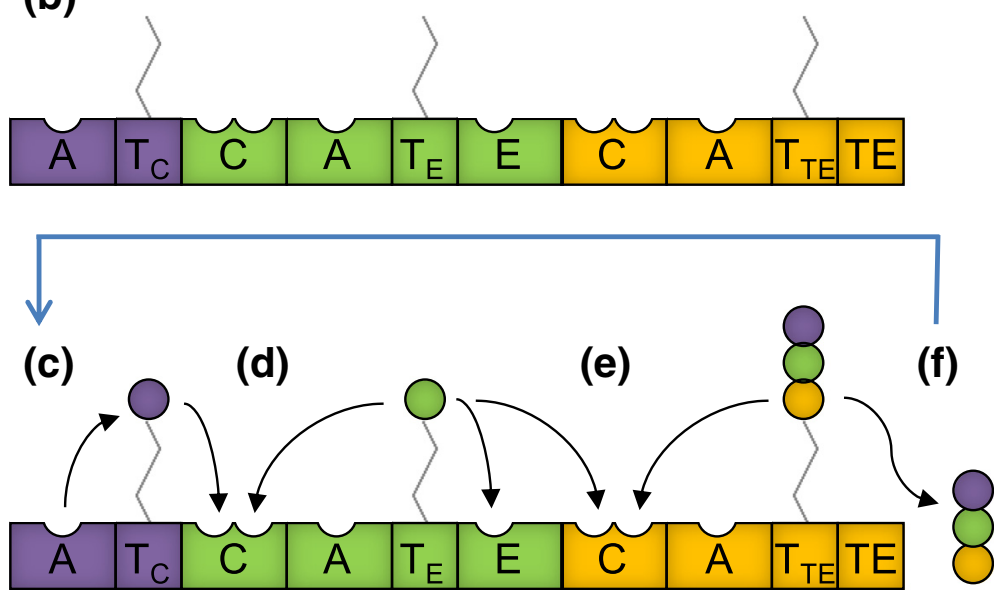

Fig. 1 NRPS modular structure and mode of peptide synthesis. A = adenylation domain (substrate recognition and activation), $T=$ thiolation domain (carrier protein), $\mathrm{C}=$ condensation domain (peptide bond formation), $\mathrm{E}=$ epimerisation domain (substrate racemisation), $T E=$ thioesterase domain (product release). Most T domains are located immediately downstream of an A domain, but they can be located upstream of three different domain types. To indicate the downstream domain, the T domains in this figure are distinguished by an additional subscript label; e.g., the $T$ domain of the initiation module is labelled $T_{C}$ to indicate that it is located immediately upstream of a $C$ domain. $\mathbf{a}$ Domain architecture of a three module NRPS containing an initiation module (core A-T domain structure; purple), an elongation module (core C-A-T domain structure, pictured here with an additional epimerisation domain; green) and a termination module (core C-A-T-TE domain structure; orange). (b) Biosynthesis occurs in an assembly line fashion, beginning with post-translational modification of each T domain by attachment of a 4'-phosphopantetheine (4'-pp) cofactor. The 4'-pp group functions as a flexible arm to coordinate the movement of substrates between catalytic sites. (c) The A domain within each module recognises and activates (via adenylation) a specific monomer, then attaches it to the $4^{\prime}$-pp group of the adjacent T domain. (d and e) In a stepwise manner, starting at the first module, each C domain catalyses peptide bond formation between the donor substrate attached to the upstream $\mathrm{T}$ domain and the acceptor substrate attached to the downstream $\mathrm{T}$ domain. Prior to this condensation reaction, each $T$ domain may need to interact with additional tailoring domains, such as the $E$ domain pictured in module 2 , which can modify the substrate borne by each module. (f) When the peptide chain reaches the termination module, the product is released by a thioesterase (TE) domain via hydrolysis or intramolecular cyclisation. The cycle of synthesis may then repeat to generate many copies of the same peptide 
synonymous A domain substitutions into PvdD (a bimodular NRPS from Pseudomonas aeruginosa PAO1 that incorporates two L-threonine residues at the C-terminus of the peptide siderophore pyoverdine [14]) yielded recombinant enzymes that were highly active in vivo, whereas nine out of nine non-synonymous A domain substitutions were inactive $[11,12]$. The $100 \%$ success rate of the synonymous A domain substitutions suggests that disruption of native $\mathrm{C} / \mathrm{A}$ or $\mathrm{A} / \mathrm{T}$ domain interfaces was not a major factor restricting our ability to generate functional recombinant NRPS enzymes.

To overcome $\mathrm{C}$ domain proof-reading constraints, we next attempted to substitute cognate C-A domain partners into the second module of PvdD. This strategy successfully yielded two modified pyoverdine products, with $\mathrm{L}$-serine or L-lysine in place of the terminal L-threonine present in the native pyoverdine. However, six of the eight C-A domain substituted constructs we generated were inactive, including two bearing threoninespecifying A domains that had previously been active when the A domain was substituted alone [12]. We proposed that one contributing factor to the inactivity of these six constructs might be an inability of the newlyintroduced $\mathrm{C}$ domain to communicate effectively with the upstream $\mathrm{T}$ domain of the first PvdD module. Consistent with this proposal, other domain substitution studies have found that $\mathrm{T}$ domains upstream of $\mathrm{C}$ domains in their native context generally cannot function properly when placed upstream of E domains [15] or TE domains [16-19]. In two of the latter cases, it was further shown that $\mathrm{T}$ domain communication with the TE domain could readily be restored by point mutations introduced via error-prone PCR $[18,19]$. Moreover, there is at least one example where dipeptides were successfully created in vitro via T-C-A domain substitution [16], a strategy that - unlike C-A domain substitutions maintains native $\mathrm{T} / \mathrm{C}$ domain interactions (Fig. 2a, b). Collectively, these studies suggest that $\mathrm{T}$ domain interactions with downstream domains may be particularly specialised, and that $\mathrm{T}$ domains may therefore have limited portability during NRPS recombination experiments. To highlight this potential specialisation and distinguish different subtypes of $\mathrm{T}$ domain on the basis of their downstream partner, when relevant in this manuscript $\mathrm{T}$ domains are labelled with the type of domain immediately downstream noted in subscript (e.g., Figure 1, Fig. 2).

To evaluate the general portability of $\mathrm{T}$ domains, we again turned to the pyoverdine synthetase PvdD as a model NRPS enzyme for domain substitution experiments. This system offers the key advantages that pyoverdine is easily detectable in vivo due to the characteristic absorbance and fluorescence of its chromophore [20, 21] (Fig. 3a), that a conditional
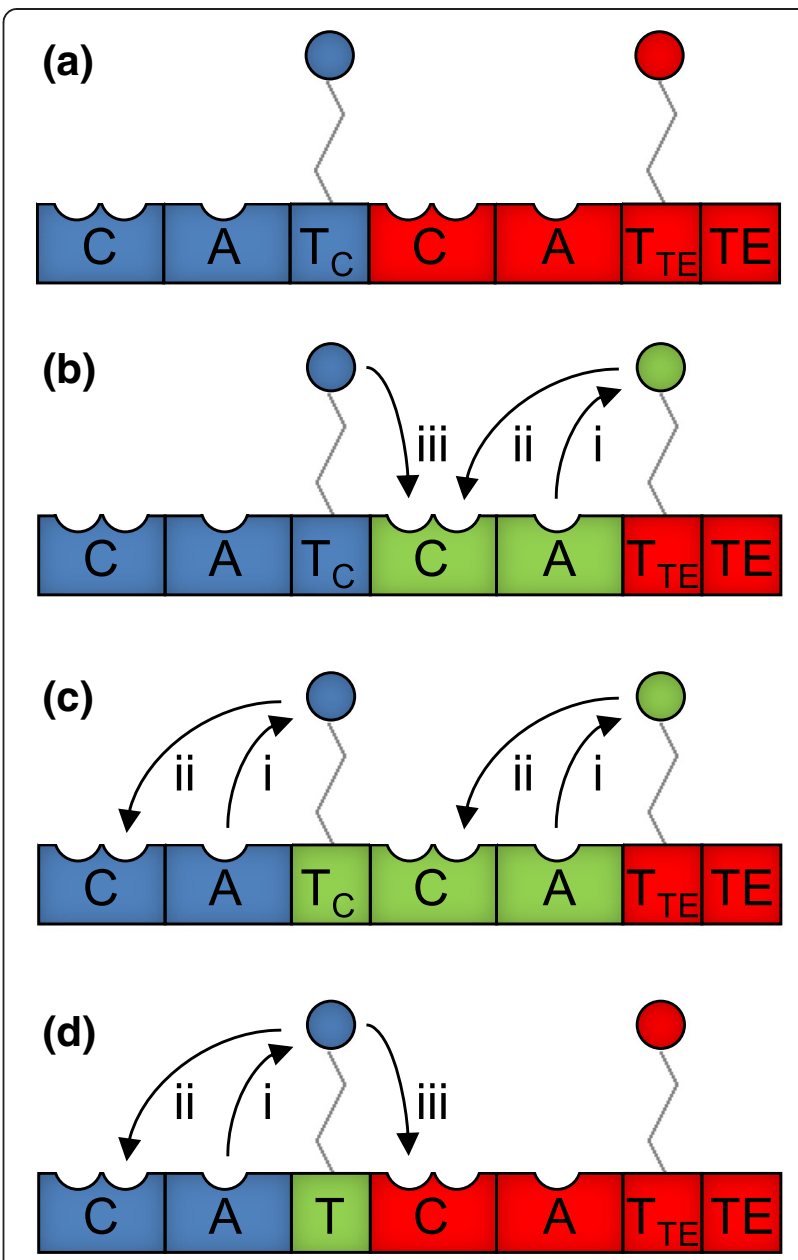

Fig. $2 \mathrm{~T}$ domain interactions that are affected by substitution of alternative domains into PvdD. a Domain architecture of PvdD with the first module shown in blue and the second module shown in red. (b-d) Alternative domains substituted into PvdD are depicted in green: (b) C-A domain substitution; (c) T-C-A domain substitution; or (d) T domain substitution. In each panel, non-cognate (i.e., potentially disrupted) interactions between $T$ domains and other domains are labelled according to the following scheme: (i) when an A domain passes a substrate to a non-cognate $T$ domain; (ii) when a $T$ domain passes a substrate to a non-cognate upstream $C$ domain; or (iii) when a $T$ domain passes a substrate to a non-cognate downstream $C$ domain. The NRPS modules from which the C-A and T-C-A domains were sourced for substitution into PvdD are those labelled Ser1, Ser2 and fhOrn 1 in Fig. 5

selection for pyoverdine synthesis can be imposed by growing the $P$. aeruginosa host in an iron-restricted environment, and that our previous work has identified recombination sites between the T-C, C-A, and A-T domain boundaries that permit effective substitution of alternative domains into PvdD [11, 12]. Furthermore, we have access in our laboratory to four different genomesequenced strains of fluorescent pseudomonads that each synthesise a different pyoverdine peptide, providing 

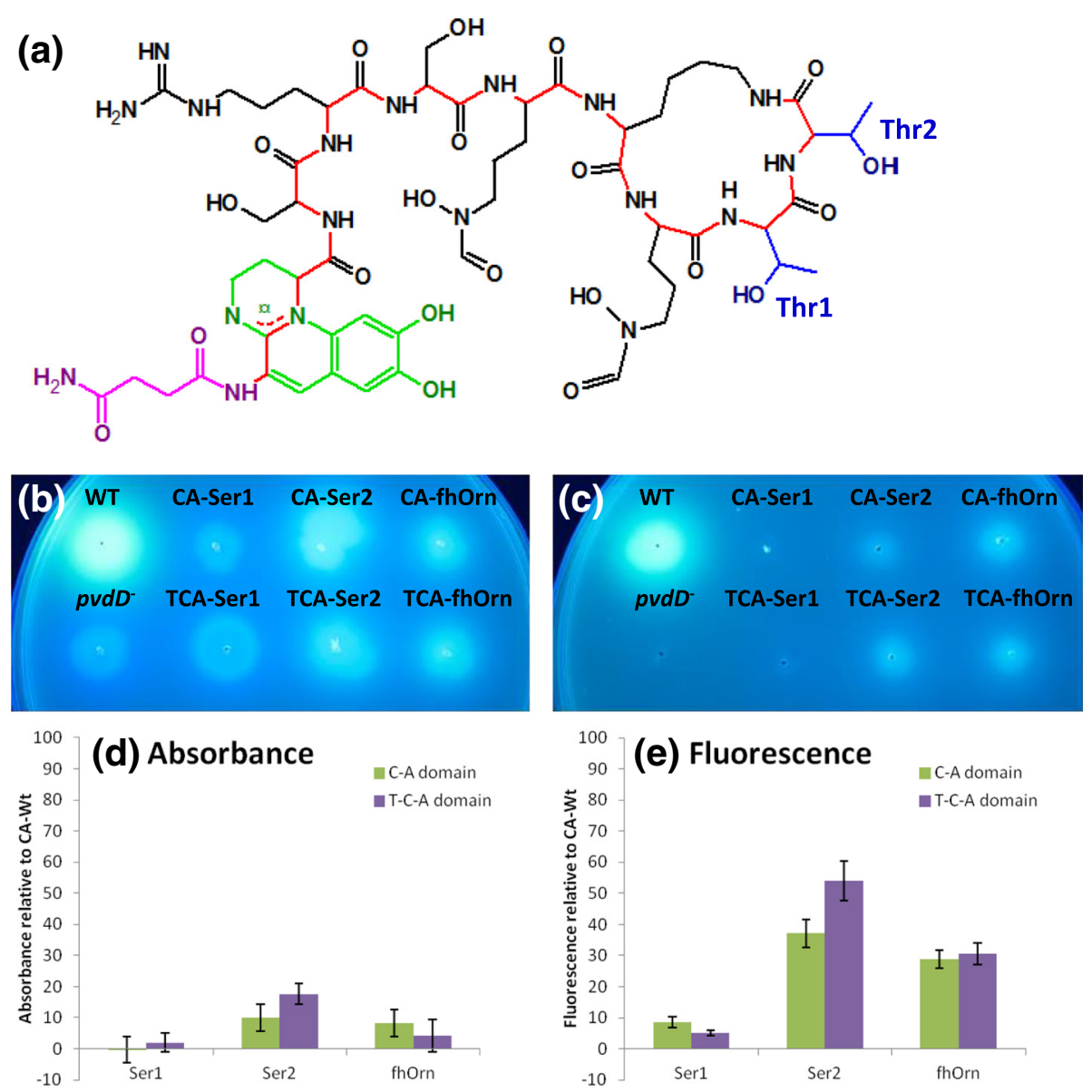

Fig. 3 Growth of C-A versus T-C-A domain substitution strains. a Structure of pyoverdine from P. aeruginosa PAO1, with the succinimide group highlighted in pink, the chromophore in green, the peptide backbone in red, and the side chains of the threonine residues incorporated by PvdD in blue. The threonine residues incorporated by modules 1 and 2 of PvdD are labelled Thr1 and Thr2, respectively. (b) and (c): Growth of C-A and T-C-A substitution strains on solid King's B media, either (b) without or (c) with addition of $200 \mu \mathrm{g} \cdot \mathrm{ml}^{-1}$ EDDHA. Plates were inoculated with the restriction site control (WT), domain substitution strains, and the $p v d D$ deletion mutant ( $p v d D^{-}$), then incubated for $24 \mathrm{~h}$ at $37^{\circ} \mathrm{C}$. Photographs were taken under UV light. (d) and (e): Pyoverdine production in liquid media from T-C-A versus C-A domain substitution strains. Values are expressed as a percentage relative to (d) the absorbance $(400 \mathrm{~nm}$ ); or (e) the fluorescence (ex. $400 \mathrm{~nm} / \mathrm{em}$. $440 \mathrm{~nm}$ ) measured for the CAThr-WT strain, having first been zeroed against the absorbance/fluorescence of the pvdD deletion mutant. Data are the mean of 6 independent replicates, and error bars indicate 1 standard deviation

a range of evolutionarily related modules for domain substitution. We used these resources to first conduct a pilot study of whether T-C-A domain substitution into PvdD might prove superior to C-A substitution as a means of generating novel pyoverdine peptides, and then performed an extensive survey of the ability of different types of $\mathrm{T}$ domain to communicate effectively with nonnative $\mathrm{C}$ domains.

\section{Results and discussion}

\section{Comparison of T-C-A and C-A domain substitution variants}

To substitute alternative domains into PvdD we used an integrating vector bearing a copy of the $p v d D$ gene that had restriction sites introduced, to enable replacement of either the native C-A domains of the second NRPS module, or the native T-C-A domains spanning the first and second modules (Fig. 2a, b). Three T-C-A domain substitution constructs were created and compared to equivalent constructs in which only the corresponding C-A domain substitutions had been made. Two of the C-A domain pairings encode a serine residue in their native context, and PvdD C-A substitution constructs bearing each of these alternative domain pairings had been analysed in our previous study [12]; in that work one (here called CA-Ser1) was non-functional and did not yield any detectable pigment, whereas the other (CA-Ser2) was found to incorporate a serine residue into pyoverdine. The third $\mathrm{C}$-A domain pairing (used to make construct CA-fhOrn) was from a N5-formyl-N5hydroxyornithine activating pyoverdine synthetase module from $P$. fluorescens SBW25, which had not previously 
been tested in domain substitution studies. The corresponding T-C-A domain substitution constructs were named TCA-Ser1, TCA-Ser2 and TCA-fhOrn.

Each C-A and T-C-A domain substitution construct was transformed into a $p v d D$ mutant strain of $P$. aeruginosa PAO1, and analysed for production of pyoverdine (structure depicted in Fig. 3a) alongside a restriction site positive control strain (CA-Wt) and an empty plasmid negative control strain $\left(p v d D^{-}\right)$. As a preliminary test for pyoverdine production, each strain was spotted onto iron-limiting King's B agar plates in either the absence
(Fig. 3b) or presence (Fig. 3c) of $200 \mu \mathrm{g} \cdot \mathrm{ml}^{-1}$ of ethylenediamine-N,N'-bis(2-hydroxyphenylacetic acid) (EDDHA; an iron-chelating agent that prevents passive uptake of iron). Of the C-A domain substitution strains, those bearing constructs CA-Ser2 and CA-fhOrn were fluorescent under UV and able to grow in the presence of EDDHA, whereas the strain containing construct CASer1 was neither fluorescent nor able to out-compete EDDHA for iron. The T-C-A domain substitution strains showed similar results to the corresponding $\mathrm{C}$-A domain substitution strains; that is, the strains bearing domain

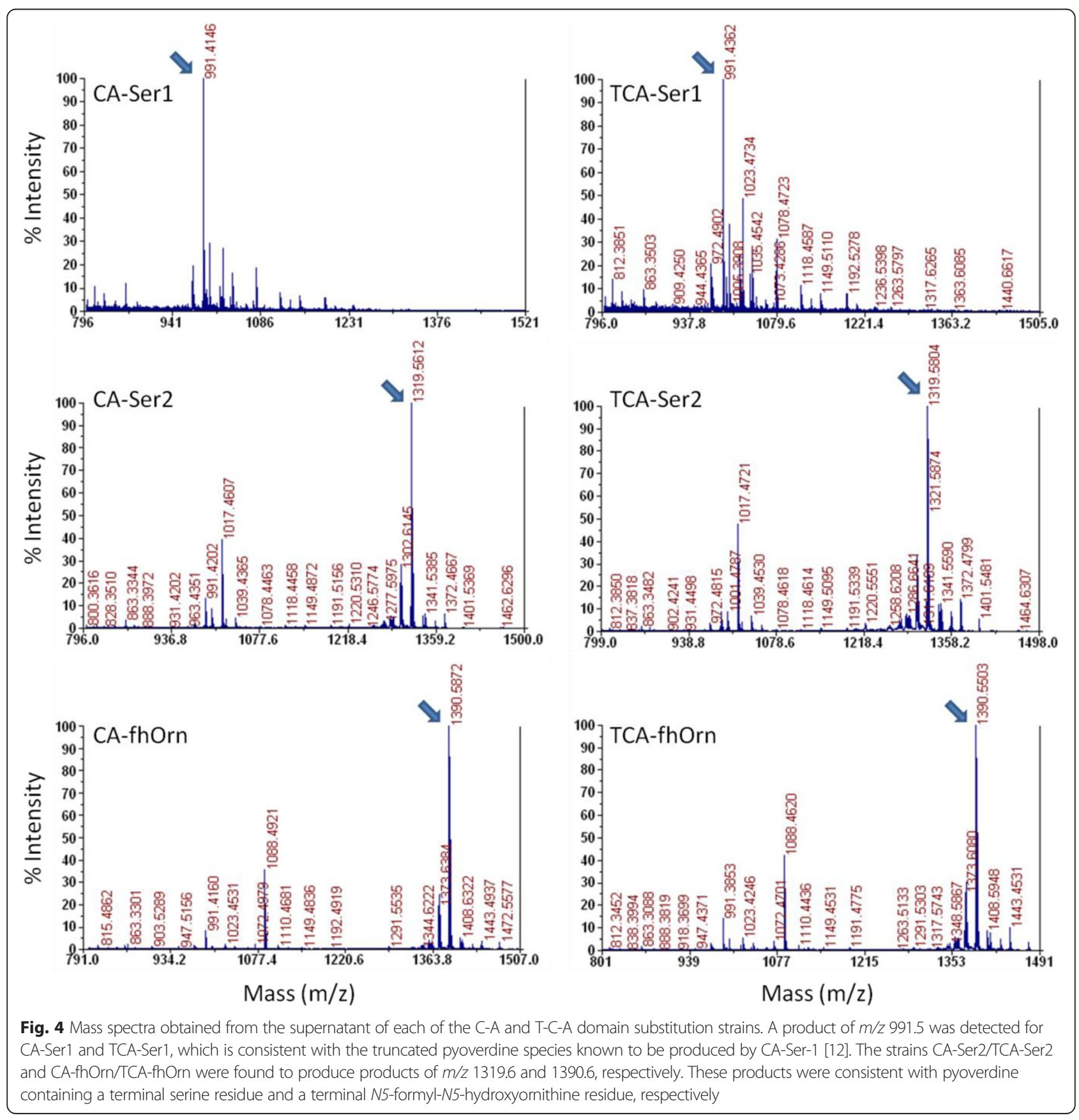


substitution constructs TCA-Ser2 and TCA-fhOrn were both fluorescent and able to grow in the presence of EDDHA, whereas the strain containing construct TCASer1 was not.

To more accurately quantify the levels of pyoverdine production, the absorbance (Fig. 3d) and fluorescence (Fig. 3e) for each T-C-A and C-A domain substitution strain was measured from the supernatant of $24 \mathrm{~h}$ cultures. As previously demonstrated, relative absorbance is a less sensitive but more linear measure of the relative amounts of pyoverdine present, whereas fluorescence is better able to detect low levels of pyoverdine [12]. By either measure, there did not appear to be a substantial difference between the C-A and T-C-A domain substitution strategies in terms of the overall yield of pigment produced.

The identity of the pyoverdine produced by each strain was then confirmed by MALDI-TOF mass spectrometry (MS) (Fig. 4). The strains CA-Ser1 and TCA-Ser1 each produced low levels of a truncated pyoverdine, a species that had previously been shown to be released by strain CA-Ser1 [12]. The other strains produced full-length pyoverdines consistent with the known substrate specificity of the substituted A domain, that is CA-Ser2 and TCA-Ser2 produced pyoverdine derived from a peptide containing a C-terminal serine residue and CA-fhOrn and TCA-fhOrn produced an entirely new pyoverdine peptide that contained a fhOrn residue in place of the terminal threonine of wild type pyoverdine. In both cases, the mass of the primary product indicated that the final pyoverdine species was still able to cyclise via peptide bond formation between the terminal carboxyl of the substituted amino acid and the amino group bound to the $\varepsilon$-carbon of an internal lysine residue.

\section{Analysis of $\mathrm{T}$ domain portability}

The lack of evidence for T-C-A domain substitution being a fundamentally superior strategy to C-A domain substitution for generating functional recombinant $\mathrm{PvdD}$ enzymes, together with a relative paucity of suitable contiguous T-C-A domain combinations available for substitution, and technical difficulties in amplifying and cloning such large DNA inserts, dissuaded us from building additional T-C-A domain substitution constructs. Instead, to focus more specifically on whether transplanted $\mathrm{T}$ domains might have difficulty in communicating effectively with non-native $C$ domains, a series of $\mathrm{T}$ domain substitutions were made by replacing the first $\mathrm{T}$ domain of PvdD according to the substitution scheme represented in Fig. 2c.

When considered in the context of altered domain interactions, $\mathrm{T}$ domain substitutions are fundamentally similar to C-A domain substitutions; that is, both establish a scenario in which there is perturbation of (i) native
$\mathrm{A} / \mathrm{T}$ domain interactions; (ii) upstream $\mathrm{C}$ and downstream $\mathrm{T}$ domain interactions; and (iii) upstream $\mathrm{T}$ and downstream $\mathrm{C}$ domain interactions (Fig. 2a, 2c). As we previously found five out of five synonymous A domain substitutions into PvdD to be active [11, 12], it can be inferred that A domains are readily able to communicate with non-native $\mathrm{T}$ domains. We therefore reasoned that our proposed $\mathrm{T}$ domain substitution strategy would shed light on whether non-native $\mathrm{T}$ and $\mathrm{C}$ domains can also communicate effectively.

Eighteen $\mathrm{T}$ domain substitution constructs were created, using a variety of $\mathrm{T}$ domains sourced from the $P$. aeruginosa $\mathrm{PAO} 1$, P. syringae 1448a, P. putida KT2440 and P. fluorescens SBW25 pyoverdine NRPS gene clusters (Fig. 5) ( $\mathrm{T}$ domain exchange boundaries and amino acid identities shared with the substituted PvdD $\mathrm{T}$ domain, as well as NRPS accession numbers, are summarised in Additional file 1: Table S1). T domains from each subtype were selected to test whether there were any clear differences in the ability of $T_{C}, T_{E}$ or $\mathrm{T}_{\mathrm{TE}}$ domains to function effectively when transplanted immediately upstream of a $\mathrm{C}$ domain. An additional nomenclature, $\mathrm{T}_{\mathrm{Ct}}$, was introduced to distinguish $\mathrm{T}_{\mathrm{C}}$ domains that act in trans with a downstream $\mathrm{C}$ domain in their native context (Fig. 5, red highlighted $\mathrm{T}$ domains) versus the more common $\mathrm{T}_{\mathrm{C}}$ domains that act in cis with a downstream $\mathrm{C}$ domain (Fig. 5, blue highlighted $\mathrm{T}$ domains). This distinction was made to allow for the possibility that $\mathrm{T}_{\mathrm{Ct}}$ domains may carry additional recognition elements, in addition to short communication domains, that allow them to associate with the correct downstream $C$ domain [22, 23]. In total, six $\mathrm{T}_{C}$ domains and four of each of $\mathrm{T}_{\mathrm{Ct}}, \mathrm{T}_{\mathrm{E}}$ and $\mathrm{T}_{\mathrm{TE}}$ domains were substituted into the first module of PvdD, giving rise to $\mathrm{T}$ domain substitution strains $\mathrm{C} 1-$ C6, Ct1-Ct4, E1-E4, and TE1-TE4 as explained in Fig. 5. The substituted regions ranged from $34.6 \%$ to $61.5 \%$ shared identity with the corresponding sequence from PvdD (Additional file 1: Table S1).

The $18 \mathrm{~T}$ domain substitution strains, together with the empty plasmid negative control strain $\left(p v d D^{-}\right)$and the restriction site positive control (T1), were spotted onto KB agar plates both with and without EDDHA (Fig. 6). Each of the $\mathrm{T}_{\mathrm{C}}$ domain substitution strains (C1-C6) were fluorescent at levels indistinguishable from control strain T1 and were all highly viable in the presence of EDDHA (Fig. 6a). In contrast, the other $\mathrm{T}$ domain substitution strains varied greatly in their fluorescence and ability to grow in the presence of EDDHA, with at least one strain for each $\mathrm{T}$ domain subtype being largely non-fluorescent on $\mathrm{KB}$ agar and greatly impaired in growth in the presence of EDDHA (Fig. 6, panels b-d).

When grown in liquid media, the levels of absorbance (Fig. 7a) and fluorescence (Fig. 7b) of supernatant from 


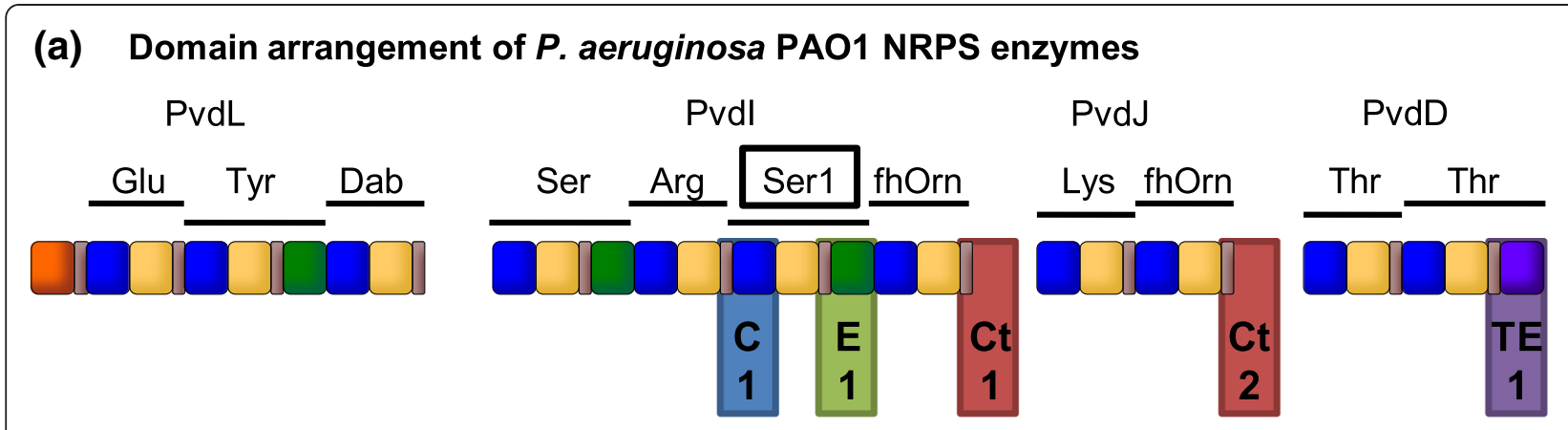

(b) Variable peptide chains from other pseudomonads

P. syringae pv. phaseolicola $1448 \mathrm{~A}$

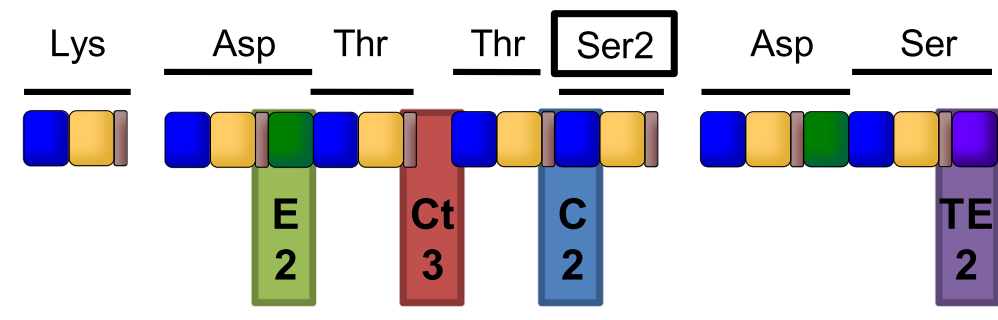

Condensation domain

P. putida KT2440
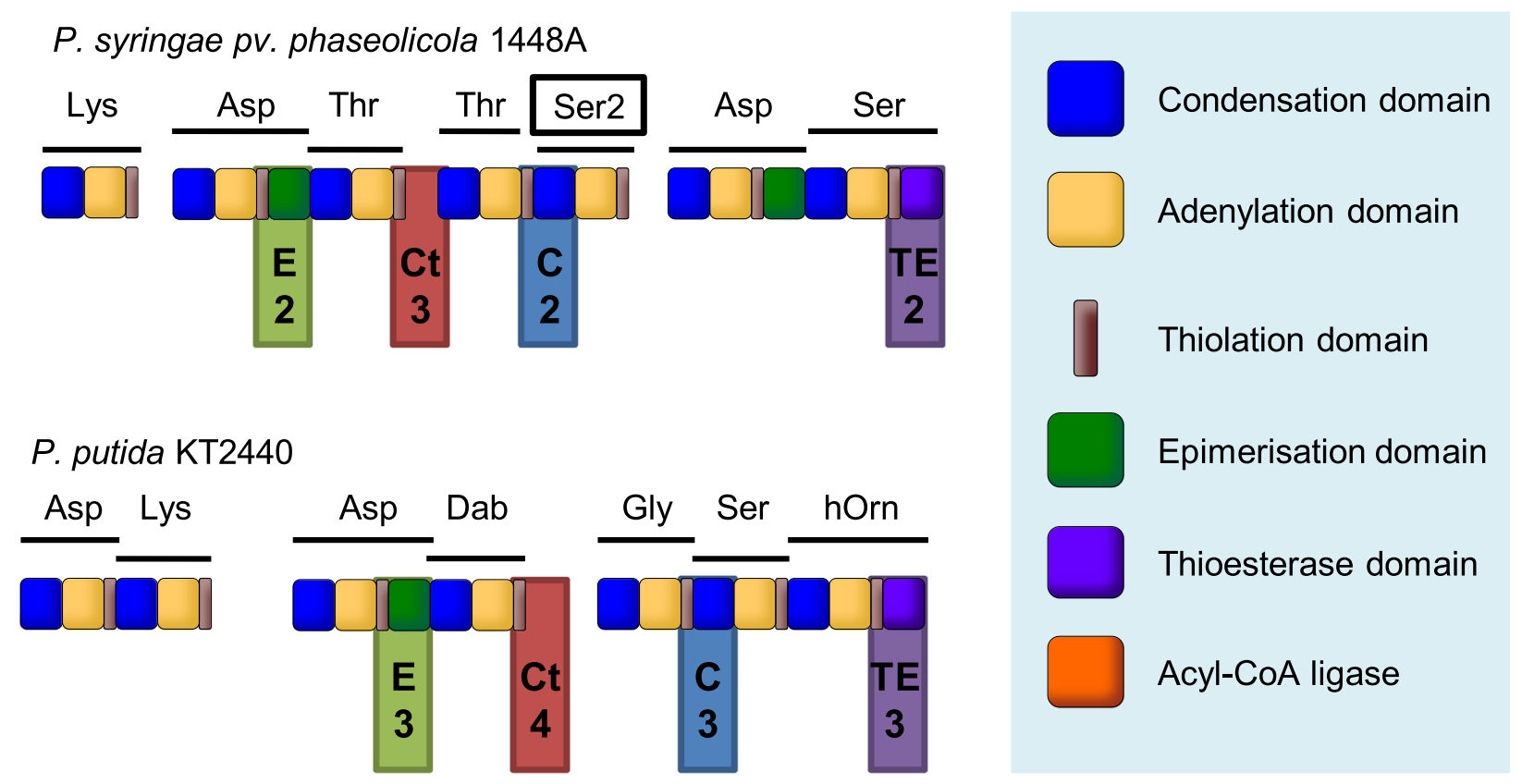

P. fluorescens SBW25

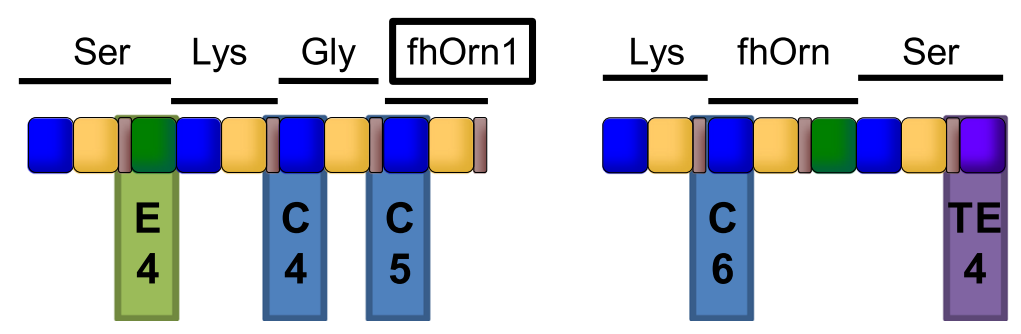

Fig. 5 Location of T domains within Pseudomonas pyoverdine NRPS gene clusters that were used to substitute the T1 domain of pvdD. a Domain architecture of the four NRPS genes involved in P. aeruginosa PAO1 pyoverdine synthesis. (b) Domain architecture of genes involved in the synthesis of pyoverdine variable peptide chains from three other Pseudomonas strains. Location and numbering of the six $T_{C}$ (blue), four $T_{C t}$ (red), four $T_{E}$ (green), and four $\mathrm{T}_{\mathrm{TE}}$ (purple) domains used for domain substitution are indicated by the colour-coded shaded boxes. Standard abbreviations are used to identify the amino acid substrate incorporated by each module, with the following additions: Dab, 2,4-diaminobutyric acid; fhOrn, L-N5-formyl-N5-hydroxyornithine; hOrn, N5-hydroxyornithine. Within this figure the three modules from which domains were sourced for C-A and T-C-A domain substitution are identified by rectangles around the name of the substrate activated by that module as well as addition of numbers after that substrate name, to correspond with the nomenclature used in the C-A and T-C-A domain substitutions 

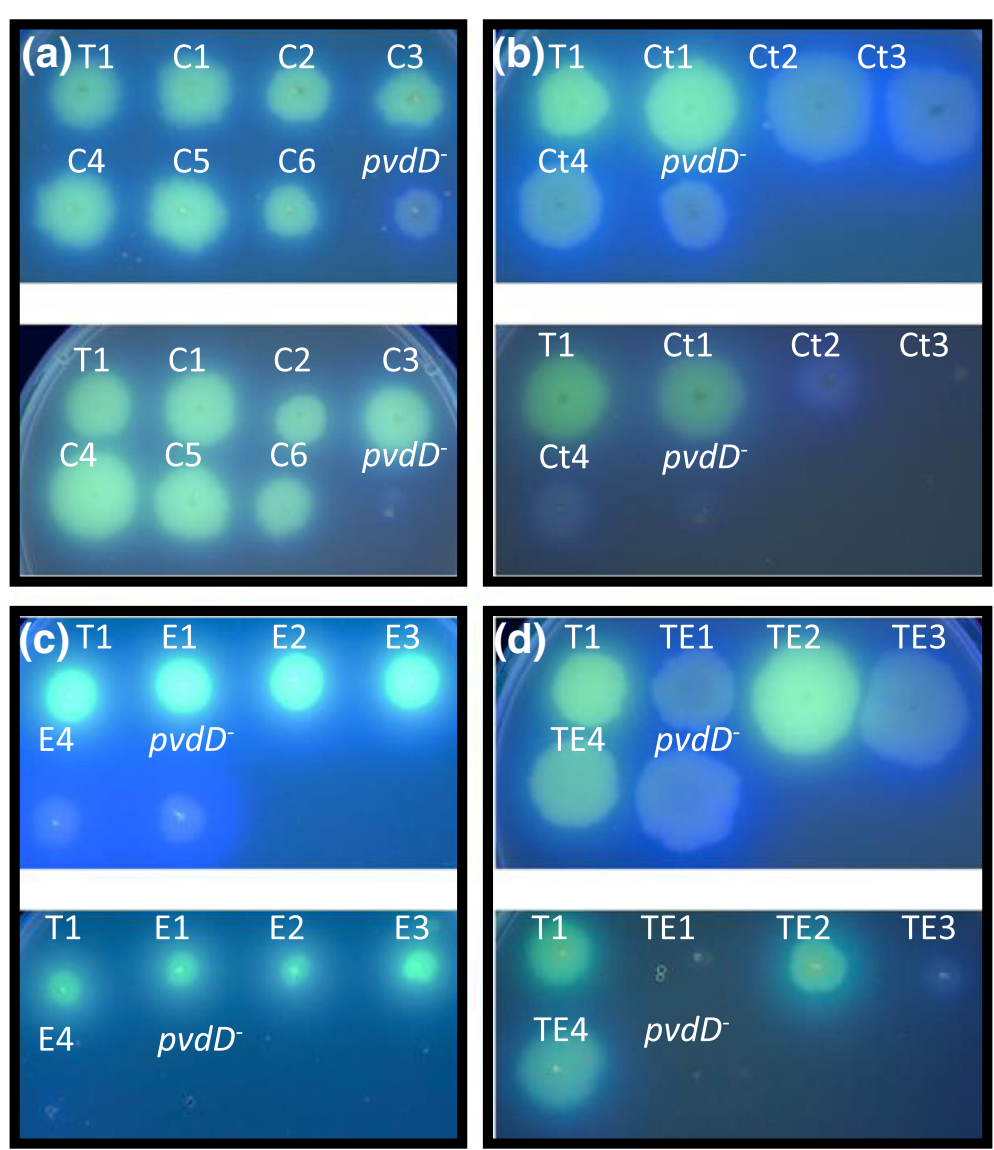

Fig. 6 Growth of $T 1$ domain substitution strains on solid media. Substitutions are grouped as a $T_{C}$ domain substitutions, (b) $T_{C t}$ domain substitutions, (c) $T_{E}$ domain substitutions or (d) $T_{T E}$ domain substitutions. For each type of substitution, the top panel is for growth on $K B$ media and the bottom figure is for growth on $\mathrm{KB}$ media supplemented with EDDHA. T1 refers to the restriction site control of the native $T_{C}$ domain ligated back into pST1; and $p v d D^{-}$is the $p v d D$ deletion mutant negative control. Plates were incubated for $24 \mathrm{~h}$ at $37^{\circ} \mathrm{C}$, and photographs were taken under UV light

cultures of each of the $\mathrm{T}$ domain substitution strains were consistent with the qualitative findings on solid media (Fig. 6). Whereas strains C1-C6 were all found to produce high levels of pyoverdine, one of each of the $\mathrm{T}_{\mathrm{Ct}}, \mathrm{T}_{\mathrm{E}}$ and $\mathrm{T}_{\mathrm{TE}}$ substitution strains appeared to make no pyoverdine at all (Ct3, E4, TE1), while others (Ct2, Ct4, TE3, TE4) were severely impaired in their level of pyoverdine production. However, the remaining $\mathrm{T}_{\mathrm{Ct}}, \mathrm{T}_{\mathrm{E}}$ and $\mathrm{T}_{\mathrm{TE}}$ substitution strains produced near wild type levels of pyoverdine.

MALDI-TOF MS was used to analyse the supernatants from cultures that exhibited limited or no apparent pyoverdine production. For the apparent non-producing strains $\mathrm{E} 4$ and TE1, no pyoverdine-like products could be detected. However, the other apparent non-producing strain $\mathrm{Ct} 3$ was detected to synthesise the same truncated $991.5 \mathrm{~m} / \mathrm{z}$ product (Fig. 8a) that had been detected in culture supernatants from the non-functional strains CA-Ser1 and TCA-Ser1 (Fig. 4). The remaining $\mathrm{T}$ domain substitution strains were all found to produce wild type pyoverdine (Fig. 8).

Thus, only 3 out of the $18 \mathrm{~T}$ domain substitution strains were completely unable to synthesise pyoverdine, none of these being strains in which the native $T_{c}$ domain of $\mathrm{PvdD}$ had been substituted by another $\mathrm{T}_{\mathrm{c}}$ domain. These data suggest that $\mathrm{T}$ domains transplanted upstream of a nonnative $C$ domain generally retain functionality, contrasting with earlier studies in which $\mathrm{T}_{\mathrm{C}}$ domains placed upstream of non-native E [15] or TE [16-19] domains were severely impaired or inactive. Our data also confirm that the boundaries we selected between the PvdD A-T and T-C domains were appropriate recombination sites for $\mathrm{T}$ domain substitution. This is an important point; in one other previous study it was found that the $\mathrm{T}_{\mathrm{TE}}$ domain from the single module indigoidine synthetase BpsA from Streptomyces lavendulae was unable to substitute for the $\mathrm{T}_{\mathrm{TE}}$ domain of the closely related single module indigoidine synthetase IndC from Photorhabdus luminescens until optimal recombination sites were identified [24]. 


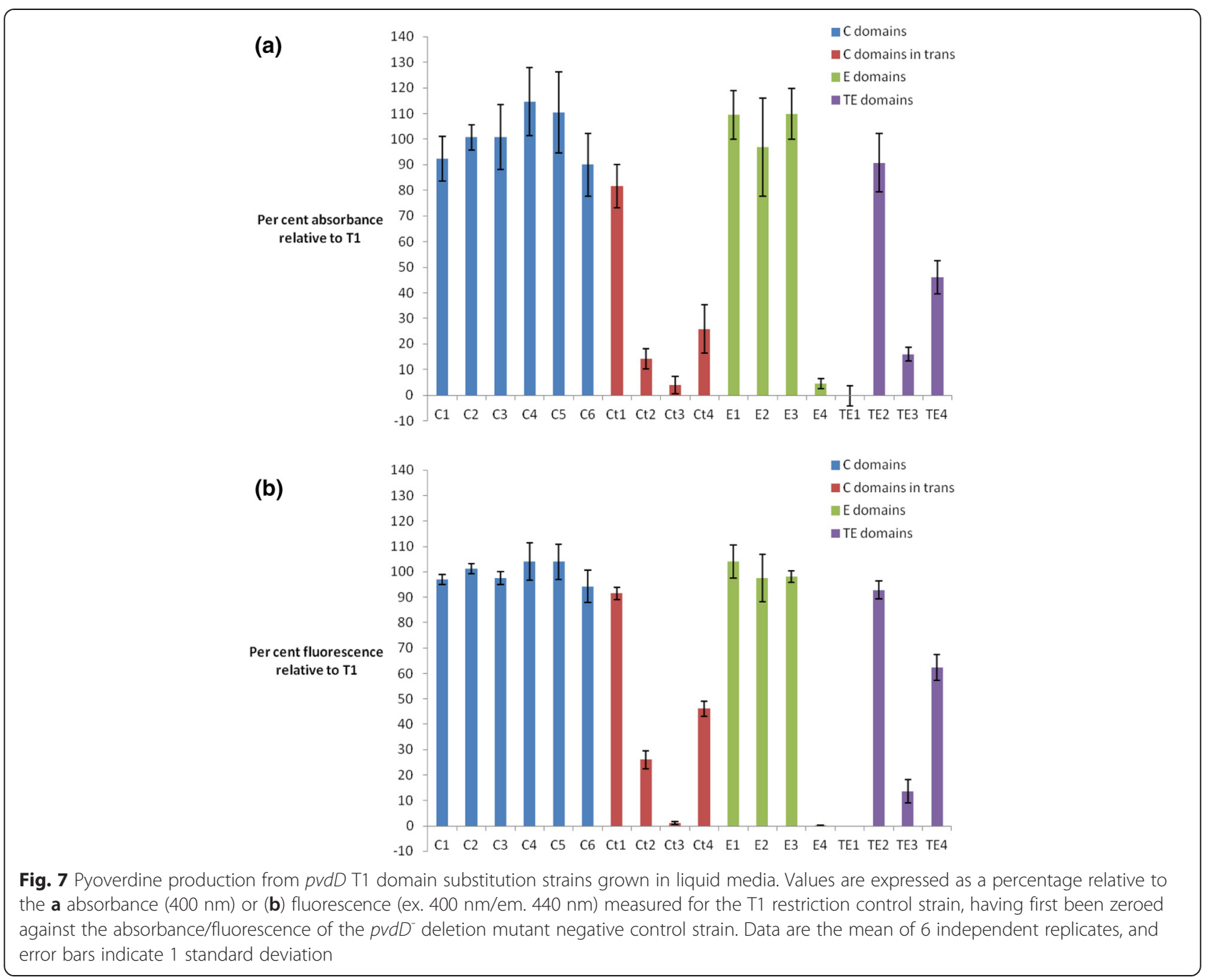

\section{Conclusions}

In previous work we found that a majority of C-A domain substitutions in the second module of PvdD yielded inactive recombinant enzymes [12]. We hypothesised that this might stem from the $\mathrm{T}$ domain of the first PvdD module being unable to communicate effectively with the newly-introduced $\mathrm{C}$ domain. If this hypothesis were true, then T-C-A domain substitutions would be expected to prove superior to C-A domain substitutions as a means of generating novel non-ribosomal peptide products. Although T-C-A domain substitution has previously been used to successfully create a bi-modular recombinant NRPS that was functional in vitro [16], the authors did not construct the equivalent C-A domain substitution construct to compare relative activities. Here, while we were able to use T-C-A domain substitution to successfully generate an entirely new pyoverdine species that had fhOrn at the C-terminus, and as an alternative means of producing a serine-substituted pyoverdine we had previously generated via $\mathrm{C}$-A domain substitution [12], we did not observe substantial differences between the T-C-A substitution and C-A domain substitution strategies in terms of product yield.

We also found that $\mathrm{T}$ domains were generally tolerant of being relocated immediately upstream of the $\mathrm{C}$ domain of the terminal PvdD module, particularly if the introduced $\mathrm{T}$ domain had been located immediately upstream of a $\mathrm{C}$ domain in its native context. That is, within their new environment they were generally capable of interacting effectively with non-native $\mathrm{C}$ and $\mathrm{A}$ domains upstream, and a non-native $\mathrm{C}$ domain downstream. This capability appeared to be independent of the amino acid identity shared between the introduced $\mathrm{T}$ domains and the PvdD $\mathrm{T}$ domain that they were replacing. For example, the $\mathrm{T}_{\mathrm{Ct}}, \mathrm{T}_{\mathrm{E}}$ and $\mathrm{T}_{\mathrm{TE}}$ domains that shared the highest sequence identity with the PvdD $T$ domain were actually the least active when substituted into PvdD (Additional file 1: Table S1). We consider this general portability of $\mathrm{T}$ domains to locations upstream of $\mathrm{C}$ domains to be quite remarkable, in light of the 


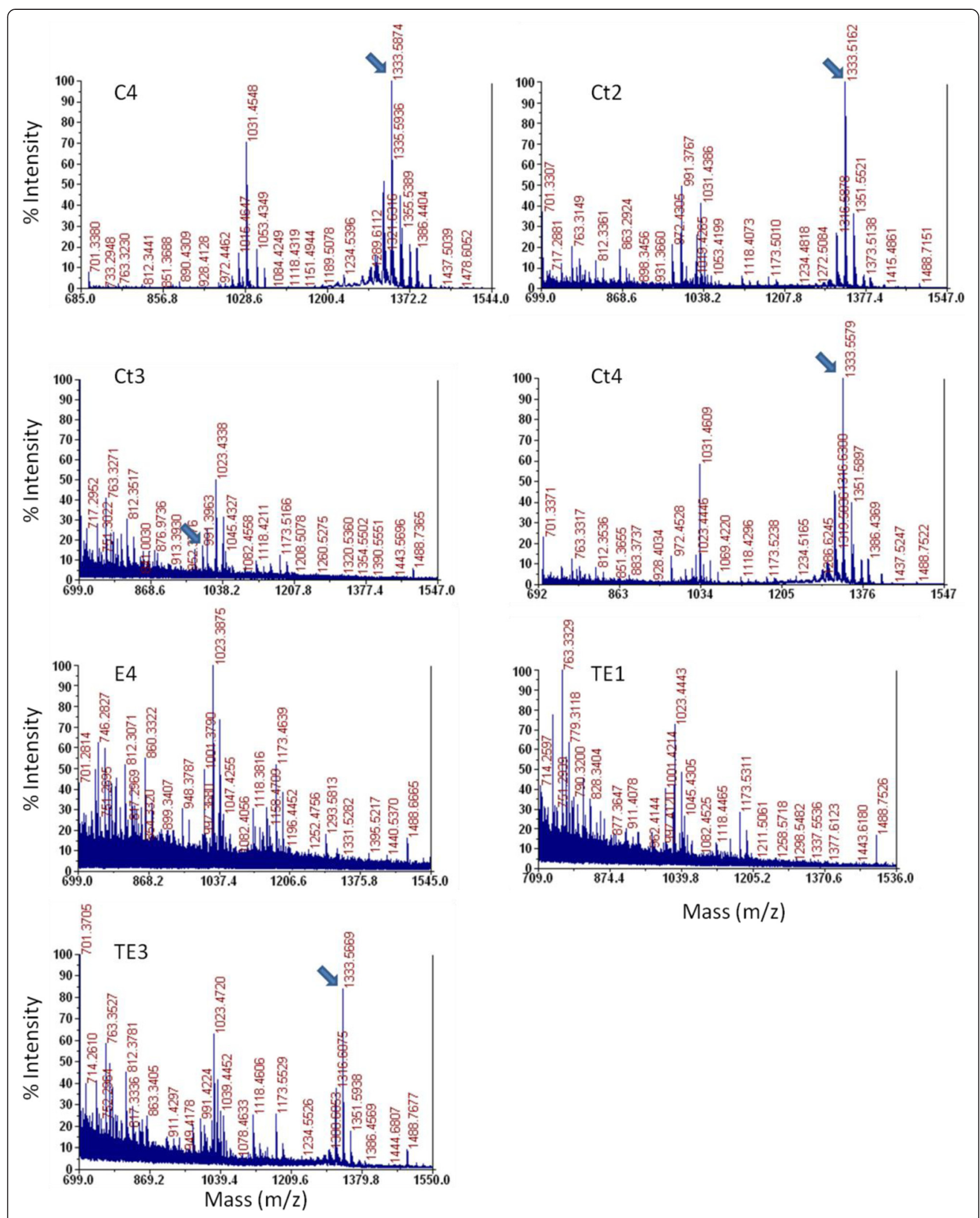

Fig. 8 Mass spectra obtained from the supernatant of each of the T1 domain substitution strains. Arrows indicate peaks at 991.5 or $1333.6 \mathrm{~m} / \mathrm{z}$, corresponding to the masses of the previously detected truncated and wild-type pyoverdine species, respectively 
extreme conformational and functional demands that are placed on $\mathrm{T}$ domains [13, 25-27], as well as previous studies indicating that $\mathrm{T}_{\mathrm{C}}$ domains are not generally portable upstream of E or TE domains [15-19]. From a biotechnology perspective it is a promising finding, as it suggests that $\mathrm{T}$ domain functional incompatibility is unlikely to be a major driver of recombinant NRPS inactivity following C-A or T-C-A domain substitution.

Collectively, our data suggest that the low success rates of our previous C-A domain substitutions into module 2 of PvdD [12] were not due to inability of the newly introduced $\mathrm{C}$ domains to communicate effectively with the PvdD module $1 \mathrm{~T}$ domain. Rather, we propose that the loss of function may have been due to inability of the introduced $\mathrm{C}$ domain to receive the large incoming pyoverdine peptide at its donor site, possibly due to steric constraints. The original work of Belshaw et al., which demonstrated no side-chain amino acid specificity at the donor site of $\mathrm{C}$ domains, used only single amino acids as donor substrates [9]. In contrast, later work by Stein et al. identified specificity within the $\mathrm{C}$ domain donor site for some dipeptides [28]. Similarly, Clugston et al. [29] observed stereo specificity at the $C$ domain donor site towards a tetrapeptide, but not towards the single amino acid substrate. The studies by Stein et al. [28] and Clugston et al. [29] strongly suggest that the size and composition of the incoming donor peptide can influence activity. Thus, steric constraints at the donor site position might explain the variable success rate of $C$ domain substitutions, with some $\mathrm{C}$ domains being more tolerant of the new peptide chain. Ultimately, this would mean that both $\mathrm{A}$ and $\mathrm{C}$-A domain substitution strategies are impeded by $\mathrm{C}$ domain limitations - the former by acceptor site selectivity, and the latter by donor site constraints. Overall, a greater understanding of $\mathrm{C}$ domain specificity is needed as it may aid in future efforts to perform functional NRPS domain substitutions.

\section{Methods}

\section{Bacterial strains and growth conditions}

E. coli $\mathrm{DH} 5 \alpha, P$. aeruginosa $\mathrm{PAO} 1$ and $P$. putida KT2440 were sourced from existing Ackerley lab stocks. P. syringae pv. phaseolicola 1448A was generously provided by Prof. John Mansfield (Imperial College, London), and P. fluorescens SBW25 by Prof. Paul Rainey (Massey University, Auckland, NZ). All strains were grown in LB media with shaking at $200 \mathrm{rpm}$, at $28{ }^{\circ} \mathrm{C}$ for $P$. syringae pv. phaseolicola $1448 \mathrm{~A}$ and $37{ }^{\circ} \mathrm{C}$ for other strains. For maintenance of plasmids, tetracycline was added to a final concentration of $15 \mu \mathrm{g} / \mathrm{ml}$ for E. coli and $100 \mu \mathrm{g} / \mathrm{ml}$ for $P$. aeruginosa PAO1. Where necessary, to prevent passive uptake of iron, EDDHA was added to a final concentration of $200 \mu \mathrm{g} \cdot \mathrm{ml}^{-1}$.

\section{General DNA methodology}

All PCR primers and plasmids used in this study are shown in Additional file 2: Tables S2 and S3, respectively. Primers were designed to amplify pyoverdine NRPS domains from the genomes of fluorescent pseudomonads based on previous annotation by Owen and Ackerley [30] (P. syringae 1448a), Moon et al. [31] ( $P$. fluorescens SBW25), and Ravel and Cornelis [32] (P. putida KT2440) as well as the NRPS analysis tools available online (http://nrps.igs.umaryland.edu/nrps/; [33]). PCR reactions used Phusion ${ }^{\mathrm{Tm}}$ DNA polymerase (Finnzymes; Espoo, Finland). Plasmids created in this work were sequence verified by Macrogen Inc. (Seoul, South Korea).

\section{C-A and T-C-A domain substitution}

$\mathrm{C}-\mathrm{A}$ and T-C-A domain substitution plasmids were constructed from pSW196 [34] in E. coli DH5 $\alpha$. C-A domain constructs were created as previously described [8], with the primers CA-fhOrn_Fwd and CAfhOrn_Rev used to amplify the new C-A domain pairing. To generate the T-C-A domain substitution plasmid pTCA, the C-A domains from the first module of $p v d D$ were PCR amplified using primers CATfwd and CAWt_Rev and ligated into pSW196 using the NotI and SacI restriction sites. The reverse primer introduced a NotI site in exactly the same location that was used for A domain substitutions previously [11]. Then, the T-TE domains from the second module of $p v d D$ were introduced into the construct as previously described for the C-A domain substitution plasmid [12]. Target T-C-A domains were then PCR amplified using the corresponding primer sets listed in Additional file 2: Table S2, and introduced into the substitution plasmids via NotI restriction digest and ligation. Correct orientation of the introduced T-C-A domain amplicons was confirmed by sequencing. The resulting plasmids were then transformed into P. aeruginosa PAO1 for single-copy integration at the attB locus [35].

\section{T domain substitution}

The domain substitution plasmid pST1 was derived from the plasmid pTCA. The second module of $p v d D$ was amplified using the primers CATTE_Fwd and TTeRev. Next the PCR product and PTCA vector were each digested with NotI and SacI, and the PCR product was ligated into pTCA. This replaced the T-Te domains of pTCA with the PCR amplified second module of $p v d D$. The primer CATTE_Fwd had added NotI and SpeI restriction sites immediately upstream of the $\mathrm{C}$ domain of the second module of $p v d D$, which allowed the subsequent insertion of $\mathrm{T}$ domains into these restriction sites. $\mathrm{T}$ domains for substitution into pST1 were amplified from the genomes of different fluorescent pseudomonads as indicated in Fig. 5 
and Additional file 1: Table S1, using the primers listed in Additional file 2: Table S2 (primers being named according to the $\mathrm{T}$ domain they amplify, with $\mathrm{F}$ indicating a forward primer, and $\mathrm{R}$ a reverse primer; e.g., the primers for creating strain $\mathrm{C} 1$ are named $\mathrm{C} 1 \mathrm{~F}$ and $\mathrm{C} 1 \mathrm{R})$. As per the $\mathrm{C}-\mathrm{A}$ and T-C-A domain substitutions, the $\mathrm{T}$ domain substitution plasmids were transformed into P.aeruginosa PAO1.

\section{Measurement of pyoverdine production}

For measuring pyoverdine levels in liquid media, strains were grown in $200 \mu \mathrm{L}$ of LB in a 96 well plate for $24 \mathrm{~h}$ at $37^{\circ} \mathrm{C}$. This starter culture was used to inoculate M9 media containing $0.2 \%(\mathrm{w} / \mathrm{v})$ L-arabinose and $4 \mathrm{~g} / \mathrm{l}$ succinate $(\mathrm{pH} 7.0)$ at a $20 \mathrm{x}$ dilution and total volume of $200 \mu \mathrm{L}$. After $24 \mathrm{~h}$ of growth, cultures were centrifuged to pellet bacteria, and then $100 \mu \mathrm{L}$ of supernatant transferred to a fresh 96 well plate. Supernatant was diluted $2 \mathrm{x}$ in fresh M9 media to give a total volume of $200 \mu \mathrm{L}$. Fluorescence (ex. $400 \mathrm{~nm} / \mathrm{em}$. $440 \mathrm{~nm})$ and absorbance $(400 \mathrm{~nm})$ were measured using an EnSpire 2300 Multilabel Reader (Perkin Elmer, Waltham, MA, USA).

\section{Mass spectrometry}

Cultures were grown according to the methods for measurement of pyoverdine production, above. Cells were pelleted by centrifugation and a sample of supernatant was mixed with matrix solution $(500 \mu \mathrm{L}$ acetonitrile, $500 \mu \mathrm{L}$ ultrapure water, $1 \mu \mathrm{L}$ trifluoroacetic acid, $10 \mu \mathrm{g} \alpha$-Cyano-4-hydroxycinnamic acid) in a volumetric sample to matrix ratio ranging from 1:5 to 1:20. Aliquots of $1 \mu \mathrm{l}$ from each sample were spotted in duplicate onto an Opti-TOF 384 well MALDI plate (Applied Biosystems, Foster City, CA) and allowed to dry at room temperature. Spots were analyzed using a MALDI TOF/ TOF 5800 mass spectrometer (Applied Biosystems) in positive ion mode. Each spot was externally calibrated using cal2 calibration mixture (Applied Biosystems). Peaks in spectra were labelled in Data Explorer (Applied Biosystems).

\section{Availability of supporting data}

The data sets supporting the results of this article are included within the article and its additional files (Additional file 1: Tables S1, Additional file 2: Table S2, Additional file 3: Table S3).

\section{Additional files}

Additional file 1: Supplementary Table S1. Proteins used for T domain substitutions in this study and percent identity shared between the substituted region and the corresponding sequence from PvdD. (DOCX $19 \mathrm{~kb})$
Additional file 2: Supplementary Table S2. Oligonucleotide primers used in this study. (DOCX $21 \mathrm{~kb}$ )

Additional file 3: Supplementary Table S3. Plasmids used in this study. (DOCX $20 \mathrm{~kb}$ )

Competing interests

The authors declare that they have no competing interests.

\section{Authors' contributions}

MC carried out all experimental work. DA obtained funding to support this research. DA and MC jointly conceived of the study, participated in its design and drafted the manuscript. All authors read and approved the final manuscript.

\section{Acknowledgements}

We thank Associate Professor Bill Jordan at the Victoria University of Wellington Centre for Biodiscovery for access to the Proteomics Facility; and Professor lain Lamont at Otago University for his generous gift of the EDDHA used in this study. This work was supported by the Royal Society of New Zealand Marsden Fund [contract number VUW0901] and the Victoria University Research Fund.

\section{Author details}

${ }^{1}$ School of Biological Sciences, Victoria University of Wellington, Wellington, New Zealand. ${ }^{2}$ Centre for Biodiscovery, Victoria University of Wellington, Wellington, New Zealand. ${ }^{3}$ Maurice Wilkins Centre for Molecular Biodiscovery, School of Biological Sciences, University of Auckland, Auckland, New Zealand.

Received: 1 March 2015 Accepted: 28 July 2015

Published online: 13 August 2015

\section{References}

1. Caboche $S$, Leclere V, Pupin M, Kucherov G, Jacques P. Diversity of Monomers in Nonribosomal Peptides: towards the Prediction of Origin and Biological Activity. J Bacteriol. 2010;192:5143-50.

2. Felnagle EA, Jackson EE, Chan YA, Podevels AM, Berti AD, McMahon MD, et al. Nonribosomal Peptide Synthetases Involved in the Production of Medically Relevant Natural Products. Mol Pharm. 2008;5:191-211.

3. Stein T, Vater J, Kruft V, Otto A, Wittmann-Liebold B, Franke P, et al. The Multiple Carrier Model of Nonribosomal Peptide Biosynthesis at Modular Multienzymatic Templates. J Biol Chem. 1996;271:15428-35.

4. Hur GH, Vickery CR, Burkart MD. Explorations of catalytic domains in non-ribosomal peptide synthetase enzymology. Nat Prod Rep. 2012;29:1074-98.

5. Marahiel MA, Stachelhaus T, Mootz HD. Modular Peptide Synthetases Involved in Nonribosomal Peptide Synthesis. Chem Rev. 1997;97:2651-74.

6. Sieber SA, Marahiel MA. Molecular Mechanisms Underlying Nonribosomal Peptide Synthesis: Approaches to New Antibiotics. Chem Rev. 2005;105:715-38.

7. Stevens BW, Joska TM, Anderson AC. Progress toward re-engineering non-ribosomal peptide synthetase proteins: a potential new source of pharmacological agents. Drug Dev Res. 2006;66:9-18.

8. Calcott MJ, Ackerley DF. Genetic manipulation of non-ribosomal peptide synthetases to generate novel bioactive peptide products. Biotechnol Lett. 2014;37:383-9.

9. Belshaw PJ, Walsh CT, Stachelhaus T. Aminoacyl-CoAs as Probes of Condensation Domain Selectivity in Nonribosomal Peptide Synthesis. Science. 1999:284:486-9.

10. Ehmann DE, Trauger JW, Stachelhaus T, Walsh CT. Aminoacyl-SNACs as small-molecule substrates for the condensation domains of nonribosomal peptide synthetases. Chem Biol. 2000;7:765-72.

11. Ackerley DF, Lamont IL. Characterization and Genetic Manipulation of Peptide Synthetases in Pseudomonas aeruginosa PAO1 in Order to Generate Novel Pyoverdines. Chem Biol. 2004;11:971-80.

12. Calcott MJ, Owen JG, Lamont IL, Ackerley DF. Biosynthesis of Novel Pyoverdines by Domain Substitution in a Nonribosomal Peptide Synthetase of Pseudomonas aeruginosa. Appl Environ Microbiol. 2014:80:5723-31.

13. Tanovic A, Samel SA, Essen L-O, Marahiel MA. Crystal Structure of the Termination Module of a Nonribosomal Peptide Synthetase. Science. 2008;321:659-63 
14. Ackerley DF, Caradoc-Davies TT, Lamont IL. Substrate specificity of the nonribosomal peptide synthetase PvdD from Pseudomonas aeruginosa. J Bacteriol. 2003;185:2848-55.

15. Linne U, Doekel S, Marahiel MA. Portability of Epimerization Domain and Role of Peptidyl Carrier Protein on Epimerization Activity in Nonribosomal Peptide Synthetases. Biochemistry (Mosc). 2001;40:15824-34.

16. Doekel S, Marahiel MA. Dipeptide formation on engineered hybrid peptide synthetases. Chem Biol. 2000;7:373-84.

17. Duerfahrt T, Doekel S, Sonke T, Quaedflieg PJLM, Marahiel MA. Construction of hybrid peptide synthetases for the production of a-I-aspartyl-|phenylalanine, a precursor for the high-intensity sweetener aspartame. Eur J Biochem. 2003;270:4555-63.

18. Owen JG, Robins KJ, Parachin NS, Ackerley DF. A functional screen for recovery of 4'-phosphopantetheinyl transferase and associated natural product biosynthesis genes from metagenome libraries. Environ Microbiol. 2012;14:1198-209.

19. Zhou Z, Lai JR, Walsh CT. Directed evolution of aryl carrier proteins in the enterobactin synthetase. Proc Natl Acad Sci U S A. 2007;104:11621-6.

20. Hannauer M, Yeterian E, Martin LW, Lamont IL, Schalk IJ. An efflux pump is involved in secretion of newly synthesized siderophore by Pseudomonas aeruginosa. FEBS Lett. 2010;584:4751-5.

21. Yeterian E, Martin LW, Guillon L, Journet L, Lamont IL, Schalk IJ. Synthesis of the siderophore pyoverdine in Pseudomonas aeruginosa involves a periplasmic maturation. Amino Acids. 2009:38:1447-59.

22. Chiocchini C, Linne U, Stachelhaus T. In Vivo Biocombinatorial Synthesis of Lipopeptides by COM Domain-Mediated Reprogramming of the Surfactin Biosynthetic Complex. Chem Biol. 2006;13:899-908.

23. Koglin A, Walsh CT. Structural insights into nonribosomal peptide enzymatic assembly lines. Nat Prod Rep. 2009;26:987.

24. Beer $\mathrm{R}$, Herbst $K$, Ignatiadis $N$, Kats I, Adlung L, Meyer $H$, et al. Creating functional engineered variants of the single-module non-ribosomal peptide synthetase IndC by T domain exchange. Mol Biosyst. 2014;10:1709-18.

25. Koglin A, Mofid MR, Löhr F, Schäfer B, Rogov W, Blum M-M, et al. Conformational Switches Modulate Protein Interactions in Peptide Antibiotic Synthetases. Science. 2006;312:273-6.

26. Frueh DP, Arthanari $H$, Koglin A, Vosburg DA, Bennett AE, Walsh CT, et al. Dynamic thiolation-thioesterase structure of a non-ribosomal peptide synthetase. Nature. 2008;454:903-6.

27. Samel SA, Schoenafinger G, Knappe TA, Marahiel MA, Essen L-O. Structural and Functional Insights into a Peptide Bond-Forming Bidomain from a Nonribosomal Peptide Synthetase. Structure. 2007;15:781-92.

28. Stein DB, Linne U, Marahiel MA. Utility of epimerization domains for the redesign of nonribosomal peptide synthetases. FEBS J. 2005;272:4506-20.

29. Clugston SL, Sieber SA, Marahiel MA, Walsh CT. Chirality of Peptide BondForming Condensation Domains in Nonribosomal Peptide Synthetases: The C5 Domain of Tyrocidine Synthetase Is a DCL Catalyst. Biochemistry (Mosc). 2003:42:12095-104

30. Owen JG, Ackerley DF. Characterization of pyoverdine and achromobactin in Pseudomonas syringae pv. phaseolicola 1448a. BMC Microbiol. 2011;11:218.

31. Moon C, Zhang X-X, Matthijs S, Schafer M, Budzikiewicz H, Rainey P. Genomic, genetic and structural analysis of pyoverdine-mediated iron acquisition in the plant growth-promoting bacterium Pseudomonas fluorescens SBW25. BMC Microbiol. 2008:8:7.

32. Ravel J, Cornelis P. Genomics of pyoverdine-mediated iron uptake in pseudomonads. Trends Microbiol. 2003;11:195-200.

33. Bachmann BO, Ravel J. 2009. Chapter 8 Methods for In Silico Prediction of Microbial Polyketide and Nonribosomal Peptide Biosynthetic Pathways from DNA Sequence Data, p. 181-217. In David A. Hopwood (ed.), Methods in Enzymology. Academic Press. doi:10.1016/S0076-6879(09)04808-3

34. Baynham PJ, Ramsey DM, Gvozdyev BV, Cordonnier EM, Wozniak DJ. The Pseudomonas aeruginosa Ribbon-Helix-Helix DNA-Binding Protein AlgZ (AmrZ) Controls Twitching Motility and Biogenesis of Type IV Pili. J Bacteriol. 2006;188:132-40.

35. Wang Z, Xiong G, Lutz F. Site-specific integration of the phage ФCTX genome into the Pseudomonas aeruginosa chromosome: characterization of the functional integrase gene located close to and upstream of attP. Mol Gen Genet MGG. 1995:246:72-9.

\section{Submit your next manuscript to BioMed Central and take full advantage of:}

- Convenient online submission

- Thorough peer review

- No space constraints or color figure charges

- Immediate publication on acceptance

- Inclusion in PubMed, CAS, Scopus and Google Scholar

- Research which is freely available for redistribution 